\title{
Most Findings Obtained With Untimed Visual Illusions Are Confounded
}

\author{
Paola Bressan (iD) and Peter Kramer \\ Dipartimento di Psicologia Generale, University of Padova, Italy
}

\section{Significance}

Our eyes deceive us. The moon looks bigger near the horizon than up in the sky, and white up in the sky but dark gray under an astronaut's boot. Such illusions not only help uncover how we perceive reality but also are used to investigate cognitive styles, mental disorders, and cultural differences. For example, certain illusions appear weaker to patients with schizophrenia, or to remote tribespeople as opposed to urbanites. Here we show for the first time that, as we look at them longer, illusions can change in counterintuitive ways: some decrease, some remain the same, and others increase. Differences in how individuals perceive illusions are typically attributed to inequalities in their mental processes or capacity. Yet we demonstrate that these differences may, instead, simply be due to differences in the time taken to inspect the illusions - which does happen to vary, randomly or systematically, across individuals and groups. Thus, some well-established disparities in how people see illusions (and the world more broadly) may themselves be illusory.

\begin{abstract}
Visual illusions have been studied extensively but their time course has not. Here we show, on a sample of over 550 people, that unrestricted presentation time (as opposed to one second) weakens the Ebbinghaus illusion but strengthens double-increment, and does not alter doubledecrement, lightness contrast. When presentation time is unrestricted, these illusions are affected in the same way (decrease, increase, no change) by how long observers look at them. Our results imply that differences in illusion magnitude between individuals or groups are confounded with differences in inspection time, no matter whether stimuli are evaluated in matching, adjustment, or untimed comparison tasks. We offer an explanation for why these three illusions progress differently - and spell out how our findings challenge theories of lightness, theories of global-local processing, and the interpretation of all research that has investigated visual illusions, or used them as tools, without considering inspection time.
\end{abstract}

\section{Keywords}

visual perception, cognitive style, perceptual style, methodological confounds, observation time, global-local processing, lightness perception, Ebbinghaus illusion

This is the accepted pre-publication version of the published article:

Bressan, P., \& Kramer, P. (2021). Most findings obtained with untimed visual illusions are confounded. Psychological Science. First published July 9, 2021. doi: 10.1177/0956797621994268

\section{Corresponding Author:}

Paola Bressan or Peter Kramer, Dipartimento di Psicologia Generale, Università di Padova, Via Venezia 8, 35131 Padova, Italy. E-mail: paola.bressan@unipd.it or peter.kramer@unipd.it 


\section{Introduction}

It takes a little bit of time to build up an image of what surrounds us, and a tad more to make sense of it (Hegdé, 2008; Ögmen \& Breitmeyer, 2006; Piaget, 1969; van Zoest et al., 2010). In the interpretation of details, taking into account the rest of the scene tends to produce remarkable benefits but also minor inconveniences, such as visual illusions. These emerge very quicklytypically within a few tens of milliseconds - and some of them continue to develop over the next several hundreds, suggesting an increasingly sophisticated integration between the element that is being judged and its context (de Brouwer et al., 2014; Schmidt \& Haberkamp, 2016; van Zoest \& Hunt, 2011). Indeed, some size effects - such as the Ebbinghaus (Schmidt et al., 2016) and Delboeuf (Oyama \& Morikawa, 1985) illusions-actually reverse when seen too briefly for contextual integration to take place.

While appreciable attention has been paid to the "microgenesis" of visual illusions - that is, their progress on extremely short time scales - no studies have explored how illusions unfold over exposure times of several seconds (the only exception being, as far as we know, Bressan \& Kramer, 2013). Yet one may expect that longer inspection times influence the observer's opportunity to attentionally discard, or disengage from, the task-irrelevant context that induces the illusion in the first place.

In the Ebbinghaus illusion, for example, a disk surrounded by small contextual disks looks larger than a physically identical disk surrounded by large ones (Figure 1, left panel). To appreciate that the two central disks are actually the same size, one ought to compare them directly and disregard the context. This should be easier to do when one inspects the figure at length rather than for less than a second or so (see also Hegdé, 2008); and the illusion does, in fact, diminish over the course of several seconds (Bressan \& Kramer, 2013).

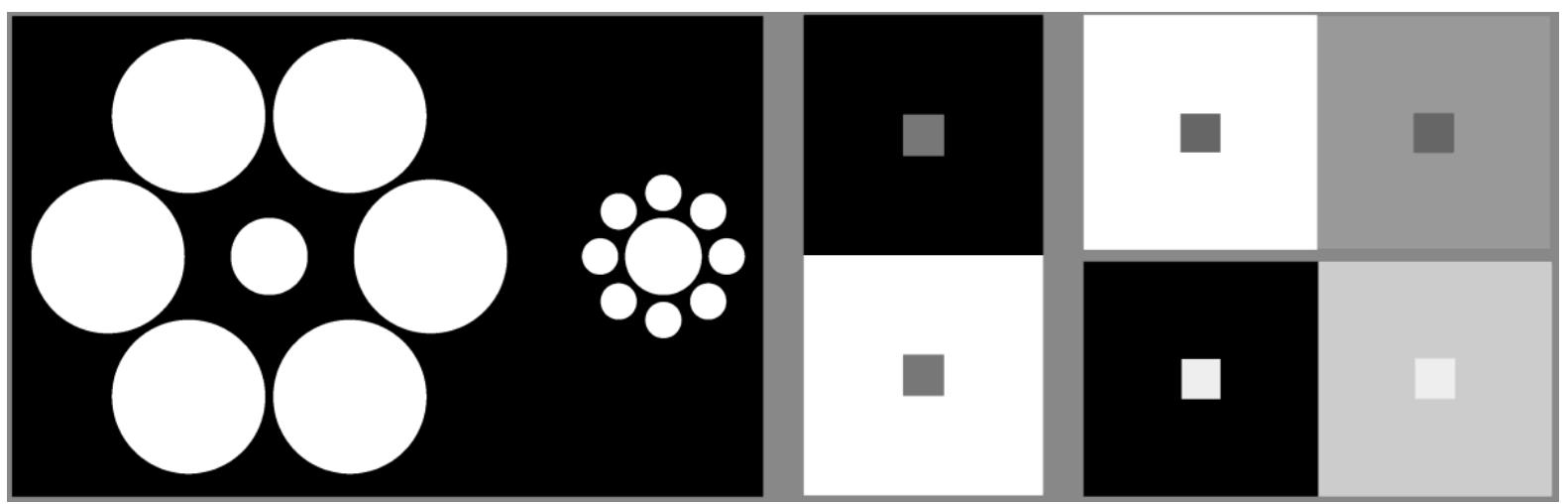

Fig. 1. Left panel: Ebbinghaus illusion. The central disk surrounded by large contextual disks looks smaller than the physically identical disk surrounded by small contextual ones. Middle panel: Lightness contrast illusion. The small square on black looks lighter than the physically identical square on white. Right panel, top: Double-decrement contrast illusion. The two identical small squares are both dimmer than their backgrounds (they are luminance decrements); the one on white looks slightly darker than the one on gray. Right panel, bottom: Double-increment contrast illusion. The two identical small squares are both brighter than their backgrounds (they are luminance increments); the one on black looks slightly lighter than the one on gray.

Now consider classical lightness contrast (Figure 1, middle panel). The Ebbinghaus illusions emerges after the other parts of the scene have been taken into account (Schmidt et al., 2016), but lightness contrast kicks in before it. Basic lightness contrast (think of a target on a plain background) peaks at the shortest possible duration, a flash of 10 milliseconds, when only the luminance 
difference along the target's edge has been processed. It then proceeds to fall rapidly over the next $150 \mathrm{~ms}$ or so, as cues from more distant edges - which help figuring out what is going on - become available as well (Kaneko \& Murakami, 2012; see also Kaneko et al., 2018; Robinson \& de Sa, 2008). Once these first $150 \mathrm{~ms}$ are past, there is not much one can do to improve lightness "accuracy". If the two central squares are small enough, simply focusing on them is not enough to insulate them from their surrounds: one cannot get truly away from the edges, and the edges are defined by the luminance contrast with the surrounds themselves. If context-dependent effects weaken with time because the context can be discounted better and better, then, we should not expect the same of illusions like these. Things could indeed go the opposite way, because the longer one inspects the central squares, the longer one inevitably ends up looking at their edges too.

The way illusions change over several seconds of inspection can pose major challenges to existing theories. Perhaps less obviously, the time course of illusions carries methodological implications for studies that measure illusion strengths by having participants either adjust a stimulus feature until it matches another (Chouinard et al., 2016; Grzeczkowski et al., 2018; Ropar \& Mitchell, 1999) or choose a match on a scale (Gilchrist et al., 1999). The same goes for research that compares illusions between people who differ in sex (Phillips et al., 2004), age (Doherty et al., 2010), culture (Caparos et al., 2012; Linnell et al., 2018), political attitude (Caparos et al., 2015), autistic tendencies (Chouinard et al., 2016; Walter et al., 2009), or mental health (Bölte et al., 2007; Happé, 1996; Hoy et al., 2004; Ropar \& Mitchell, 1999; Silverstein et al., 2015; Tibber et al., 2013). From the susceptibility to illusions shown by individuals or groups, the studies mentioned above (and many others besides) drew conclusions about processing styles or deficits. Yet all such studies had flexible inspection times and may thus have confounded an actual difference (or similarity) in illusion strength between participants with a-systematic or unsystematic - difference in the time these participants took to arrive at their judgment about the illusion.

As a case in point, Bressan and Kramer (2013) previously reported that the Ebbinghaus illusion diminishes with a particular aspect of schizotypal tendency. Their analysis, however, also revealed that the effect was entirely mediated by judgment time: the stronger their schizotypal tendency, the longer participants took to adjust one central disk until it matched the other - and it was this longer time that weakened the illusion, not the schizotypal tendency itself.

In that study, inspection time coincided with the time participants took to adjust one of the central disks by dragging a cursor along a slide. It is thus possible that the observed effects of time were in fact driven, or heavily influenced, by the fine motor control needed to perform the adjustment task. Here, we simplify the participants' critical response to one of the simplest possible: a space-bar press. To make our case, we compare the Ebbinghaus illusion, which we expect to diminish with inspection time, to lightness contrast, which we expect to remain the same or even strengthen. We do not use the classical lightness contrast illusion depicted in the middle panel of Figure 1 because this is actually a combination of two effects, which we investigate separately: a strong lightening of the square on black and a slight darkening of the square on white (Bressan, 2006; Gilchrist, 2006). These two effects are not symmetrical: indeed, as we will see, how either varies with inspection time has significant implications for theories of lightness.

\section{Method}

\section{Participants}

To provide robust power and reproducibility, we ran two independent studies and enrolled as many participants as feasible - in light of the constraint that they were all unpaid volunteers, separately recruited and tested individually in the same laboratory. Most were university students of various scientific disciplines. Data were collected, by experimenters blind to the study hypotheses, over six years (Study 1: 2014-2015; Study 2: 2016-2019). A total of 263 adults (138 women and 125 men; median and mean age 22, age range 18-30 years) participated in Study 1 and a total of 290 adults 
(195 women and 95 men; median and mean age 23, age range 19-62 years) in Study 2. So, each sample included more than twice the number of participants used in Bressan \& Kramer (2013). The experimental procedures were approved by the Institutional Review Board and were in accordance with the Declaration of Helsinki (seventh revision). All participants gave written informed consent.

\section{Apparatus and stimuli}

In both studies, stimuli were presented on a calibrated Quato Perfect Color 22 CRT monitor with a 22-inch screen shielded by a custom black hood, in the same dark or dimly lit laboratory. We used a pairwise-comparison paradigm with a fixed stimulus and a variable one with four possible values, and in the two studies we presented slightly different values at the ends of each spectrumso as to expand the overall stimulus range across studies and increase measurement sensitivity.

The stimuli for the Ebbinghaus illusion (Figure 1, left panel) were two white target disks centered on adjacent black squares $(15.5 \times 15.5 \mathrm{~cm})$. One target was surrounded by large disks (diameter: $50 \mathrm{~mm}$ in Study 1 and $45 \mathrm{~mm}$ in Study 2) and the other by small ones (diameter: $12 \mathrm{~mm}$ in Study 1 and $10 \mathrm{~mm}$ in Study 2). The size of the target surrounded by large disks was fixed (diameter: $25 \mathrm{~mm}$ ). The diameter of the other target - which tends to look larger — varied and was either 21, 22, 23, or $24 \mathrm{~mm}$ in Study 1 and 20, 21, 22, or $23 \mathrm{~mm}$ in Study 2. The position (left vs. right) of the variable half of the stimulus was counterbalanced within and between participants.

The stimuli for the lightness illusions (Figure 1, right panel) were two small target squares (both $6.4 \times 6.4 \mathrm{~cm}$ ) centered on two large background squares (both $16 \times 16 \mathrm{~cm}$ ) sitting side-by-side and surrounded by a dark frame (grayscale $=55$ ). In the case of double decrements, the backgrounds were white (grayscale $=255$ ) and middle gray (grayscale $=153$ ). The grayscale value of the target on gray was fixed (102). The grayscale value of the target on white-which tends to look darkervaried and was either 117, 122, 125, or 127 in Study 1 and 123, 125, 127, or 129 in Study 2. In the case of double increments, the backgrounds were black (grayscale=0) and light gray (grayscale $=200$ in Study 1 and grayscale $=180$ in Study 2). The grayscale value of the target on gray was fixed (222). The grayscale value of the target on black-which tends to look lighter-varied and was either 205, 208, 211, or 214 in Study 1 and 211, 213, 215, or 217 in Study 2. The position (left vs. right) of the variable half of the stimulus was counterbalanced within and between participants.

\section{Procedure}

Data were collected as part of two larger studies, but the experiment reported here was always presented first. Each illusion (double-increments, double-decrements, and Ebbinghaus, in that order) was shown in two blocks of four trials each, for a total of 8 trials per illusion and 24 trials overall. Presentation time was 1 second in one block and unlimited ("ad libitum") in the other, with the order of the two blocks counterbalanced between participants. In both the 1-second and adlibitum conditions, the participants were to press the left Z-key or the right M-key if they perceived the target on respectively the left or the right to be lighter in the double-increment illusion; darker in the double-decrement illusion; or larger in the Ebbinghaus illusion. At the start of each block (1 second or ad libitum), participants were presented with the general instructions for that block and pressed the space bar to begin. Before each stimulus, they were shown a 2500-ms reminder about whether they had 1 second or unlimited inspection time.

In the 1-second block, the stimulus disappearance was followed by the instruction to press either the left or the right key. In the ad-libitum block, participants were asked to hit the space bar as soon as they had decided which target was lighter, or darker, or larger. There was no time pressure. After hitting the space bar, participants were shown the instruction to press either the left or the right key. 


\section{Results}

Illusion-consistent responses were assigned a score of 1; illusion-inconsistent ones a score of 0 . Mean illusion strength within a block was obtained by averaging across its four trials. For each adlibitum block, we also calculated the median inspection time (i.e., the time participants took to inspect the stimulus to decide which disk was larger, or which square lighter or darker, and hit the space bar). Anticipations (responses faster than $200 \mathrm{~ms}$ ) were excluded from analysis, but results were virtually the same if these were included.

Three repeated-measures ANOVAs were carried out on illusion strengths, each with a withinsubject factor of presentation time ( 1 second vs. ad libitum) and a between-subject factor of study (1 vs. 2 ). In the 1 -second condition, the Ebbinghaus illusion was larger, $F(1,550)=53.15, p<.0001$, $\eta_{\mathrm{p}}{ }^{2}=.09$; the double-increment contrast illusion smaller, $F(1,550)=24.83, p<.0001, \eta_{\mathrm{p}}{ }^{2}=.04$; and the double-decrement contrast illusion neither larger nor smaller, $F(1,550)=2.40, p=.122, \eta_{\mathrm{p}}{ }^{2}=.004$. Corresponding mean illusions are represented in Figure 2. None of these effects interacted significantly with study. (Here main effects of study are irrelevant and we do not report them; the complete set of results is provided in the analysis outputs, see Open Practices Statement.)

Ebbinghaus illusion

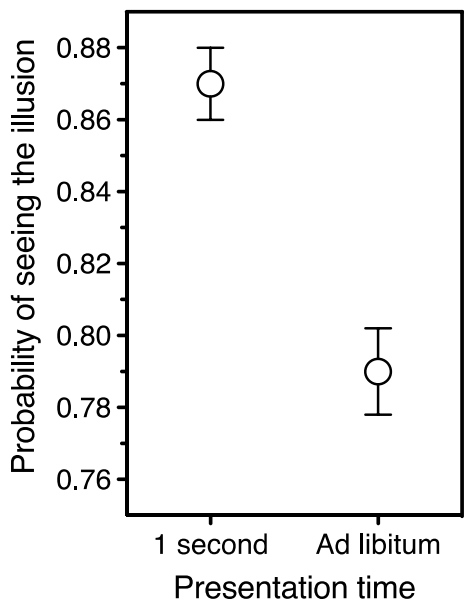

Double increments

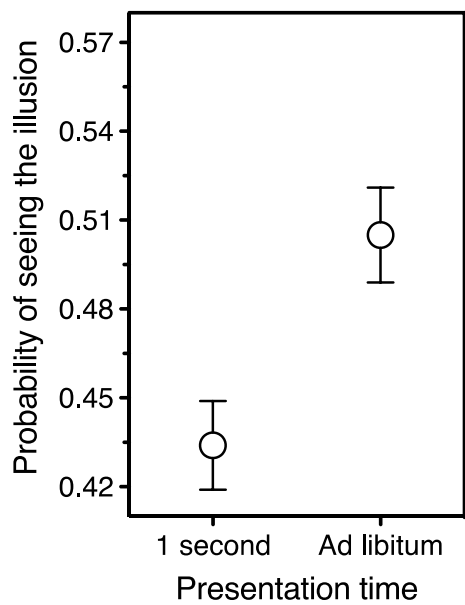

Double decrements

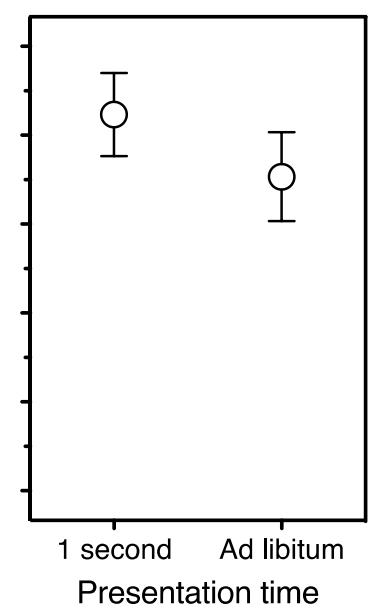

Fig. 2. Probability of seeing the illusions as a function of presentation time (1 second vs. unlimited). First panel: Ebbinghaus illusion. When presentation time is unconstrained (mean inspection time: 5.5 $\mathrm{s}, S D=4.5 \mathrm{~s}$ ), the illusion decreases. Second panel, left: lightness contrast with double increments. When presentation time is unconstrained (mean inspection time: $7.7 \mathrm{~s}, S D=5.1 \mathrm{~s}$ ), the illusion increases. Second panel, right: lightness contrast with double decrements. When presentation time is unconstrained (mean inspection time: $5.8 \mathrm{~s}, S D=3.8 \mathrm{~s}$ ), the illusion neither increases nor decreases. Error bars indicate one standard error around the mean.

Mirroring these results, Spearman correlations showed that, in the ad-libitum condition, the Ebbinghaus illusion decreased with inspection time, $r_{s}=-.45, N=552, p<.0001$; the doubleincrement illusion increased, $r_{s}=.15, N=550, p=.0003$; and the double-decrement illusion neither increased nor decreased, $r_{s}=.01, N=550, p=.749$. These results remained virtually unchanged after removing potential outliers (i.e., inspection times that deviated more than three standard deviations from the mean). Note that these findings imply that the classical lightness contrast illusion — which relies almost entirely on the perceptual lightening of the incremental target (Gilchrist, 2006) -will increase with observation time. 


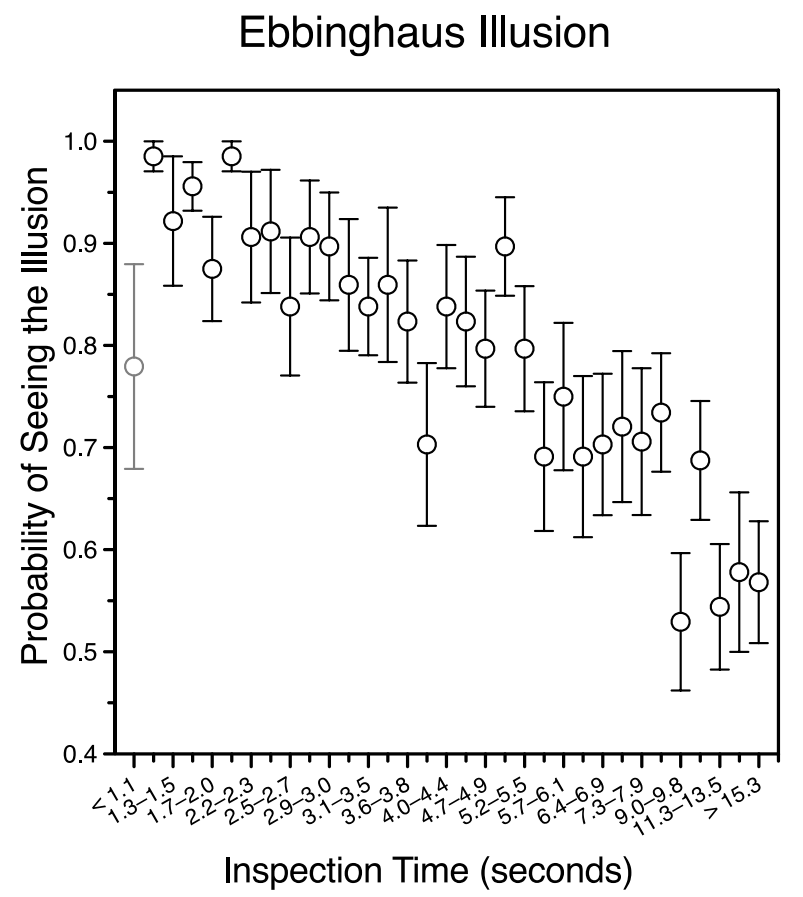

Fig. 3. Probability of seeing the Ebbinghaus illusion as a function of spontaneous inspection time, in the ad-libitum condition. Individual inspection times are grouped into 3\% bins (16-17 participants per bin, except for the last bin that includes 22), and mean illusion magnitude and standard error are plotted for each. The mean inspection time of the 17 participants in the first bin was $971 \mathrm{~ms}, S D=151 \mathrm{~ms}-$ hence shorter than in the 1-second condition, which is why the corresponding symbol is depicted in gray.

\section{Double-Increment Contrast Illusion}

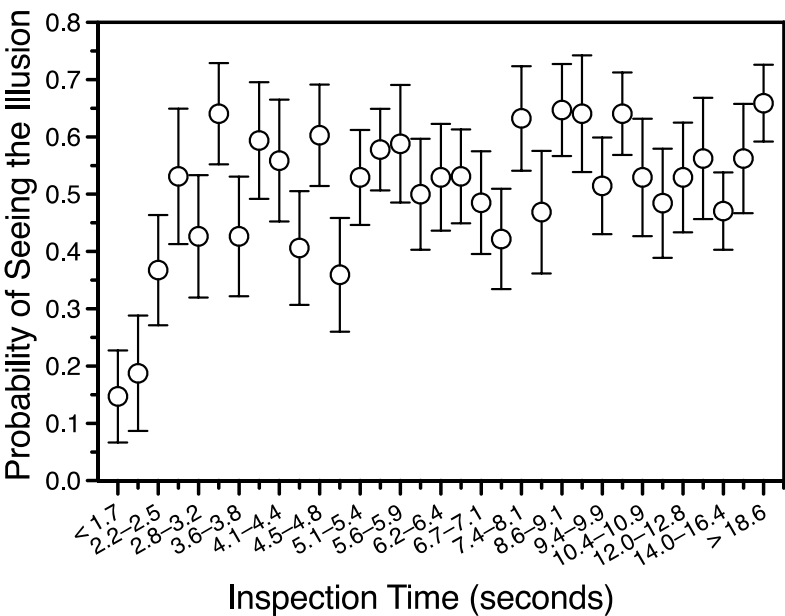

\section{Double-Decrement Contrast Illusion}

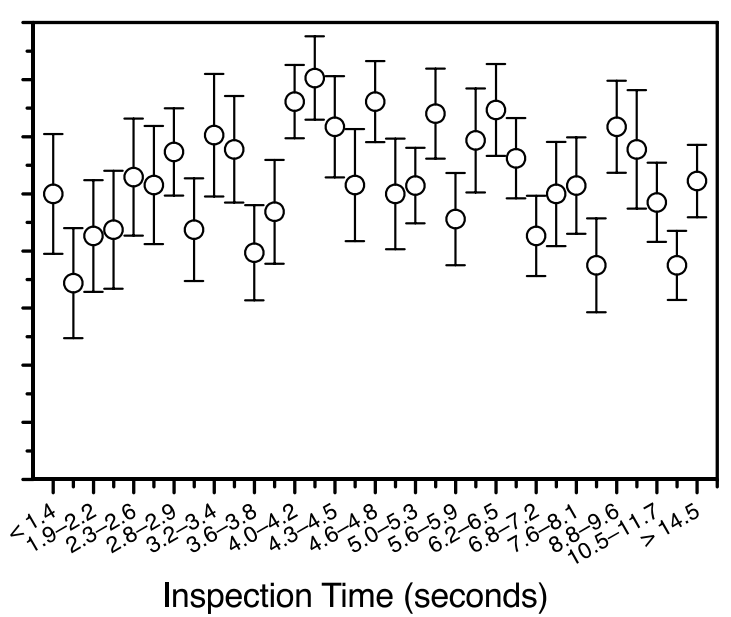

Fig. 4. Probability of seeing the double-increment (left panel) and double-decrement (right panel) lightness contrast illusions as a function of spontaneous inspection time, in the ad-libitum condition. Individual inspection times are grouped into 3\% bins (16-17 participants per bin, except for the last bin that includes 22), and mean illusion magnitude and standard error are plotted for each. Mean inspection times were longer than those in the 1-second condition for all bins including the first (double increments: $1353 \mathrm{~ms}, S D=242 \mathrm{~ms}$; double decrements: $1151 \mathrm{~ms}, S D=232 \mathrm{~ms}$ ). 
Parametric analyses showed that the best fitting curve for the decrease in the Ebbinghaus illusion with inspection time was linear, $r=.38, F(1,550)=90.92, p<.0001$, though a logarithmic fit was equally good. This result means that the Ebbinghaus illusion continued to diminish with spontaneous inspection time up to the longest one we observed, without reaching a plateau (Figure $3)$. The best fitting curve for the time course of the double-increment contrast illusion was nonlinear, with illusion strength varying with the reciprocal of inspection time, $r=.22, F(1,548)=27.68$, $p<.0001$ (outlier exclusion had virtually no effect). That is, the double-increment illusion rose steeply with time at first and then flattened out, approaching an asymptote (Figure 4, left panel). As to the double-decrement contrast illusion, no curve significantly fitted its (practically nil) progression with inspection time (Figure 4, right panel).

\section{Discussion}

The Ebbinghaus, double-increment, and double-decrement illusions respectively weakened with, strengthened with, and remained unaffected by, inspection time-whether this was constrained as opposed to unconstrained (1 second vs. unlimited) or voluntarily chosen (ad libitum). These findings bear several general methodological and theoretical implications, which we illustrate here with two notable examples: first, a reinterpretation of the role of cultural differences in global-local processing and lightness perception, and second, a better informed look at lightness-perception theories.

\section{Methodological implications: global-local processing mix-ups}

A series of well-cited studies investigated global versus local processing of visual stimuli and compared seminomadic Himba tribesmen from Namibia to British undergraduates (and other urbanites: see Caparos et al., 2020). The Ebbinghaus illusion was smaller (Caparos et al., 2012) and classical lightness contrast larger (Linnell et al., 2018) among the Himba than among the British. These effects were attributed to a stronger local (as opposed to global) bias among the former than the latter, ascribed in turn to their traditional rather than urban lifestyle. That urban experience may influence visual illusions is remarkable, particularly as regards something so very basic as lightness contrast.

Yet, because inspection time in these studies was neither controlled nor considered, a less provocative interpretation presents itself. Traditional Himba are so removed from the modern world they even lack names for geometric shapes like circles and squares (Davidoff et al., 2008). Understandably, they are also quite unaccustomed to being asked to perform exotic cognitive tasks in exchange for sugar and flour. It thus seems likely that they would find participating in such experiments more interesting than do British university students. One way in which greater engagement can express itself is more time spent on the task (Huang et al., 2012; Wise \& Kong, 2005). In reaction-time experiments, in fact, the Himba proved significantly slower, and less distractible, than British undergraduates (de Fockert et al., 2011; Linnell et al., 2013). Hence, it is perfectly possible that the Himba might also have taken longer than the British to inspect the illusions. Our own findings suggest that this would lead precisely to a weaker Ebbinghaus illusion and a stronger lightness contrast.

Three separate findings suggest that the unusual performance of the Himba may indeed be driven not by some "local bias" rooted in their intimate relationship with nature, but more simply by their greater attentional engagement. First, the Himba matched constellations of squares and crosses (after Navon, 1977) more by their local elements than by their global shapes: but such "local" choices turned out to be uncorrelated with the allegedly "local" choices they had previously made with the Ebbinghaus illusion (Caparos et al., 2012), calling into question whether these two sets of responses truly have the same cause. Second, when explicitly asked to identify either the local elements or the global shape of such constellations, the Himba outperformed British 
psychology students in both tasks (Caparos et al., 2013). Third, in reaction-time experiments that required attending a target and ignoring distractors (Linnell et al., 2013), the Himba outperformed the British when the task was relatively boring to students (determining whether an arrow points left or right), but not when it was, in the authors' very words, "engaging or interesting" to the Himba and British both (determining whether a face is Black or White).

In our present study, as well as in most of the Himba studies, optimal performance requires disregarding context, which takes effort. One can expend this effort by looking at the stimulus for longer or by concentrating on it harder. Thus, we predict that participants' motivation to respond carefully (their "response effort": Huang et al., 2012) will change visual illusions in much the same way as does spontaneous inspection time, even when such time is held constant. This implies that the strategy of matching subgroups of Himba and urbanites by their response times (as has been done, for example, to allay concerns of speed-accuracy tradeoffs in reaction-time experiments: de Fockert et al., 2011; Linnell et al., 2013) is inadequate for dismissing engagement as an explanation. And of course, while the idea that urbanization could affect visual illusions is very provocative, the idea that urbanization could affect engagement and engagement visual illusions is rather less so. Especially if "urbanization" turned out to reflect simply a different attitude of urbanized and nonurbanized people toward experiments.

\section{Theoretical implications: the timeline of grays}

Our finding that lightness contrast depends on inspection time for increments but not for decrements has important repercussions for theories of lightness. As far as we can tell, none of them is capable of accommodating this finding except the double-anchoring theory (Bressan, 2006, 2007).

The primary problem in lightness perception is that we cannot "see" the percentage of light a region reflects (its reflectance or gray shade) but only the amount of light it reflects (its luminance), which depends on both reflectance and illumination. To tell them apart we must rely on a regularity of nature: light sources tend to illuminate not only the region of interest but nearby ones too. Thus, taking into account the target's luminance relative to that of its neighbors helps discount the illumination they share. Double-anchoring theory proposes a mechanism to determine which regions are likely to be sitting under a common illumination. Within this shared-illumination framework, the target's luminance is anchored twice: once to the other regions (the target's surround) and once to the highest luminance. The target:surround and target:highest luminance ratios are then averaged. Note that both anchors are defined as white (though neither necessarily appears white in the end). Thus, anchoring establishes not only the target's relative luminance but also its specific lightness - which in the model can range from black to luminous superwhite. (On highest-luminance anchoring by itself, see also Gilchrist et al., 1999; Wallach, 1948.)

Surround anchoring is weighted relative to highest-luminance anchoring to express the surround's importance: how much information about the illuminant it is likely to be supplying and how reliable this information is. For example, a larger and more irregular surround tends to provide more information than a small, uniform one. And a bright surround (which is likely to be getting plenty of light) tends to provide more detailed, better-quality information than one that is dim (and likely to be poorly lit). In the exact same vein, a surround that is inspected for longer tends to provide more accurate and reliable information than one that is seen only very briefly.

In the model, decremental and incremental lightness contrasts are rather different animals. The double-decrement illusion (Figure 1, top right) is brought about in full by either anchoring. Surround anchoring computes the target as darker when it sits on white than on gray, because the target:surround luminance ratio is lower. Highest-luminance anchoring does that too, because-for decremental targets - the local highest luminance is the surround itself. When the relative weight of surround anchoring increases, therefore, the double-decrement illusion is expected to neither increase nor decrease. On the opposite, the double-increment illusion (Figure 1, bottom right) is entirely due to surround anchoring: the target is computed as lighter when it sits on black than on 
gray, because the target:surround luminance ratio is higher. Highest-luminance anchoring produces no illusion, because both targets are the local highest luminances and thus defined as white. When the relative weight of surround anchoring increases, therefore, the double-increment illusion is expected to increase too.

Both predictions are corroborated by evidence that whatever one does to augment the weight of the surround (for example, enlarge it or make it less homogeneous: Bressan \& Actis-Grosso, 2006; Schirillo \& Shevell, 1996) increases contrast illusions with increments but has no effect on those with decrements. Exactly the same is expected to happen if the illusion-inducing surround acquires more weight by being looked at for longer than a glimpse.

Thus, double-anchoring theory accounts effortlessly for two counterintuitive findings. First, a longer inspection time - which is bound to increase, at least over the first few seconds, the visual information available for processing - can make lightness perception less veridical rather than more. Second, this general effect entirely depends on whether the object whose color we are trying to assess is brighter or dimmer than its surroundings.

\section{Conclusion}

Much research relies on visual illusions to either build theories of them or explore differences in people's perceptual or cognitive styles. Here we have shown that the experience of visual illusions depends on how long they are looked at - in ways that are specific for each illusion and can be highly counterintuitive. Theories that cannot account for this fact are incomplete; studies that ignore it, confounded.

\section{Author Contributions}

P. Bressan supervised the project, analyzed the data, prepared the materials for open access and the figures, and laid out the implications for lightness theories. P. Kramer explored the literature and conceived the deadline experiment ( 1 second vs. ad libitum) with the Ebbinghaus illusion. The authors wrote the article together; both approved its final version for submission.

\section{ORCID iDs}

Paola Bressan (D) orcid.org/0000-0003-2763-0902

Peter Kramer id orcid.org/0000-0003-4807-7077

\section{Acknowledgments}

This work was carried out within the scope of the project "use-inspired basic research", for which the Department of General Psychology of the University of Padova has been recognized as a "Dipartimento di Eccellenza" by the Italian Ministry of University and Research. We are grateful to Massimiliano Martinelli and Luca Semenzato for programming the experiments. Data were collected by Emanuela D'Agostino and Valentina Vellani (Study 1); Lucia Tagliente, Tommaso Querci, Cecilia Marchetti, and Giulia Martorana (Study 2).

\section{Declaration of Conflicting Interests}

The authors declare that there are no conflicts of interest with respect to the authorship or the publication of this article. 


\section{Open Practices}

Neither of the studies reported in this article was formally preregistered. All the data on which the conclusions are based, along with the analysis scripts and annotated outputs, are publicly available via the Open Science Framework and can be accessed at osf.io/4ucrd.

\section{References}

Bölte, S., Holtmann, M., Poustka, F., Scheurich, A., \& Schmidt, L. (2007). Gestalt perception and local-global processing in high-functioning autism. Journal of Autism and Developmental Disorders, 37(8), 1493-1504. https://doi.org/10.1007/s10803-006-0231-x

Bressan, P. (2006). The place of white in a world of grays: A double-anchoring theory of lightness perception. Psychological Review, 113(3), 526-553. https://doi.org/10.1037/0033295X.113.3.526

Bressan, P. (2007). Dungeons, gratings, and black rooms: A defense of double-anchoring theory and a reply to Howe et al. (2007). Psychological Review, 114(4), 1111-1114. https://doi.org/10.1037/0033-295X.114.4.1111

Bressan, P., \& Actis-Grosso, R. (2006). Simultaneous lightness contrast on plain and articulated surrounds. Perception, 35(4), 445-452. https://doi.org/10.1068/p5247

Bressan, P., \& Kramer, P. (2013). The relation between cognitive-perceptual schizotypal traits and the Ebbinghaus size-illusion is mediated by judgment time. Frontiers in Psychology, 4, 343. https://doi.org/10.3389/fpsyg.2013.00343

Caparos, S., Ahmed, L., Bremner, A. J., de Fockert, J. W., Linnell, K. J., \& Davidoff, J. (2012). Exposure to an urban environment alters the local bias of a remote culture. Cognition, 122(1), 80-85. https://doi.org/10.1016/j.cognition.2011.08.013

Caparos, S., Fortier-St-Pierre, S., Gosselin, J., Blanchette, I., \& Brisson, B. (2015). The tree to the left, the forest to the right: Political attitude and perceptual bias. Cognition, 134, 155-164. https://doi.org/10.1016/j.cognition.2014.10.006

Caparos, S., Linnell, K. J., \& Blanchette, I. (2020). The local perceptual bias of a non-remote and educated population. Psychological Research, 84(5), 1211-1222. https://doi.org/10.1007/s00426-019-01158-6

Caparos, S., Linnell, K. J., Bremner, A. J., de Fockert, J. W., \& Davidoff, J. (2013). Do local and global perceptual biases tell us anything about local and global selective attention? Psychological Science, 24(2), 206-212. https://doi.org/10.1177/0956797612452569

Chouinard, P. A., Unwin, K. L., Landry, O., \& Sperandio, I. (2016). Susceptibility to optical illusions varies as a function of the Autism-Spectrum Quotient but not in ways predicted by local-global biases. Journal of Autism and Developmental Disorders, 46(6), 2224-2239. https://doi.org/10.1007/s10803-016-2753-1

Davidoff, J., Fonteneau, E., \& Fagot, J. (2008). Local and global processing: Observations from a remote culture. Cognition, 108(3), 702-709. https://doi.org/10.1016/j.cognition.2008.06.004

de Brouwer, A. J., Brenner, E., Medendorp, W. P., \& Smeets, J. B. (2014). Time course of the effect of the Müller-Lyer illusion on saccades and perceptual judgments. Journal of Vision, 14(1). https://doi.org/10.1167/14.1.4

de Fockert, J. W., Caparos, S., Linnell, K. J., \& Davidoff, J. (2011). Reduced distractibility in a remote culture. Plos One, 6(10), e26337. https://doi.org/10.1371/journal.pone.0026337

Doherty, M. J., Campbell, N. M., Tsuji, H., \& Phillips, W. A. (2010). The Ebbinghaus illusion deceives adults but not young children. Developmental Science, 13(5), 714-721. https://doi.org/10.1111/j.1467-7687.2009.00931.x

Gilchrist, A. (2006). Seeing black and white. Oxford University Press.

Gilchrist, A., Kossyfidis, C., Bonato, F., Agostini, T., Cataliotti, J., Li, X., Spehar, B., Annan, V., \& Economou, E. (1999). An anchoring theory of lightness perception. Psychological Review, 106(4), 795. https://doi.org/10.1037/0033-295X.106.4.795 
Grzeczkowski, L., Roinishvili, M., Chkonia, E., Brand, A., Mast, F. W., Herzog, M. H., \& Shaqiri, A. (2018). Is the perception of illusions abnormal in schizophrenia? Psychiatry Research, 270, 929-939. https://doi.org/10.1016/j.psychres.2018.10.063

Happé, F. G. E. (1996). Studying weak central coherence at low levels: Children with autism do not succumb to visual illusions. A research note. Journal of Child Psychology and Psychiatry, 37(7), 873-877. https://doi.org/10.1111/j.1469-7610.1996.tb01483.x

Hegdé, J. (2008). Time course of visual perception: Coarse-to-fine processing and beyond. Progress in Neurobiology, 84(4), 405-439. https://doi.org/10.1016/j.pneurobio.2007.09.001

Hoy, J. A., Hatton, C., \& Hare, D. (2004). Weak central coherence: A cross-domain phenomenon specific to autism. Autism, 8(3), 267-281. https://doi.org/10.1177/1362361304045218

Huang, J. L., Curran, P. G., Keeney, J., Poposki, E. M., \& DeShon, R. P. (2012). Detecting and deterring insufficient effort responding to surveys. Journal of Business and Psychology, 27(1), 99-114. https://doi.org/10.1007/s10869-011-9231-8

Kaneko, S., \& Murakami, I. (2012). Flashed stimulation produces strong simultaneous brightness and color contrast. Journal of Vision, 12(12). https://doi.org/10.1167/12.12.1

Kaneko, S., Murakami, I., Kuriki, I., \& Peterzell, D. H. (2018). Individual variability in simultaneous contrast for color and brightness: Small sample factor analyses reveal separate induction processes for short and long flashes. i-Perception, 9(5), 2041669518800507. https://doi.org/10.1177/2041669518800507

Linnell, K. J., Bremner, A. J., Caparos, S., Davidoff, J., \& de Fockert, J. W. (2018). Urban experience alters lightness perception. Journal of Experimental Psychology: Human Perception and Performance, 44(1), 2-6. https://doi.org/10.1037/xhp0000498

Linnell, K. J., Caparos, S., de Fockert, J. W., \& Davidoff, J. (2013). Urbanization decreases attentional engagement. Journal of Experimental Psychology: Human Perception and Performance, 39(5), 1232-1247. https://doi.org/10.1037/a0031139

Navon, D. (1977). Forest before trees: The precedence of global features in visual perception. Cognitive Psychology, 9(3), 353-383. https://doi.org/10.1016/0010-0285(77)90012-3

Ögmen, H. E., \& Breitmeyer, B. G. (Eds.). (2006). The first half second: The microgenesis and temporal dynamics of unconscious and conscious visual processes. Cambridge, MA: Mit Press.

Oyama, T., \& Morikawa, K. (1985). Temporal development of optical illusions. In J. L. McGaugh (Ed.), Contemporary psychology: Biological processes and theoretical issues (pp. 385-393). Amsterdam: North-Holland.

Phillips, W. A., Chapman, K. L. S., \& Berry, P. D. (2004). Size perception is less context-sensitive in males. Perception, 33(1), 79-86. https://doi.org/10.1068/p5110

Piaget, J. (1969). The mechanisms of perception. New York: Basic Books.

Robinson, A. E., \& de Sa, V. R. (2008). Brief presentations reveal the temporal dynamics of brightness induction and White's illusion. Vision Research, 48(22), 2370-2381. https://doi.org/10.1016/j.visres.2008.07.023

Ropar, D., \& Mitchell, P. (1999). Are individuals with autism and Asperger's syndrome susceptible to visual illusions? Journal of Child Psychology and Psychiatry, 40(8), 1283-1293. https://doi.org/10.1111/1469-7610.00544

Schirillo, J. A., \& Shevell, S. K. (1996). Brightness contrast from inhomogeneous surrounds. Vision Research, 36(12), 1783-1796. https://doi.org/10.1016/0042-6989(95)00217-0

Schmidt, F., \& Haberkamp, A. (2016). Temporal processing characteristics of the Ponzo illusion. Psychological Research, 80(2), 273-285. https://doi.org/10.1007/s00426-015-0659-8

Schmidt, F., Weber, A., \& Haberkamp, A. (2016). Dissociating early and late visual processing via the Ebbinghaus illusion. Visual Neuroscience, 33, E016.

https://doi.org/10.1017/S0952523816000134

Silverstein, S. M., Elliott, C. M., Feusner, J. D., Keane, B. P., Mikkilineni, D., Hansen, N., Hartmann, A., \& Wilhelm, S. (2015). Comparison of visual perceptual organization in 
schizophrenia and body dysmorphic disorder. Psychiatry Research, 229(1-2), 426-433. https://doi.org/10.1016/j.psychres.2015.05.107

Tibber, M. S., Anderson, E. J., Bobin, T., Antonova, E., Seabright, A., Wright, B., Carlin, P., Shergill, S. S., \& Dakin, S. C. (2013). Visual surround suppression in schizophrenia. Frontiers in Psychology, 4, 88. https://doi.org/10.3389/fpsyg.2013.00088

van Zoest, W., \& Hunt, A. R. (2011). Saccadic eye movements and perceptual judgments reveal a shared visual representation that is increasingly accurate over time. Vision Research, 51(1), 111-119. https://doi.org/10.1016/j.visres.2010.10.013

van Zoest, W., Hunt, A. R., \& Kingstone, A. (2010). Representations in visual cognition: It's about time. Current Directions in Psychological Science, 19(2), 116-120. https://doi.org/10.1177/0963721410363895

Wallach, H. (1948). Brightness constancy and the nature of achromatic colors. Journal of Experimental Psychology, 38(3), 310-324. https://doi.org/10.1037/h0053804

Walter, E., Dassonville, P., \& Bochsler, T. M. (2009). A specific autistic trait that modulates visuospatial illusion susceptibility. Journal of Autism and Developmental Disorders, 39(2), 339-349. https://doi.org/10.1007/s10803-008-0630-2

Wise, S. L., \& Kong, X. (2005). Response time effort: A new measure of examinee motivation in computer-based tests. Applied Measurement in Education, 18(2), 163-183.

https://doi.org/10.1207/s15324818ame1802_2 